\title{
Casos curiosos de embarazo ectópíco avanzado
}

\author{
Por el doctor GUILLERMO NAVAS ANGEL
}

\section{I- Embrrazos tubarios.}

El embarazo tubario o tubo-ovárico on evolución, antes del cuarto mes, no nos interesa, por cuanto entra en el conjunto, pudiéramos decir, rutinario, de la ecciesir, asaz conocido. Sin embargo, comencemos por resumir el cuadro sintomático y el acopio de sigros que son comunes a todas las variedades de ectopías ovulares.

El interrogatorio es en esta entidad tocológica de una enorme importancia, por cuanto con él, si es verídico $y$ iuicioso, se llega por lo menos a la presunción diagnóstica. Suministra la mujer afectada, datos sobre su amenorrec, diciendo que es abscluta, 0 cue, por el contrario, se ha visto matizada por episodios hemorrágicos pe. queños, o que ha estado interierida por verdaderas menstruaciones regulares o irregulares en su advenimiento, o que con las pequeñas hemorragias ha visto la expulsión de ia caduca uterina, o en fin que no ha estado en amenorrea.

Pero en esto ya comienza a influír la variedad de ecciesis por cuanto es fácil observar que las más hemorragíparas son las tubarias, fenómeno explicable porque mientras más cerca ciel útero esté la bolsa grávida, más suculenta es la caduca uterina y porque además se ha comprobado que el ovario puede suministrar un saco protector más fuerte, más elástico y de mayor duración al huevo fecundado. Si el embarazo es abdominal primitivo, bien puede faltar la hemorragia o pasar inadvertida por su discreción.

Según Conill, la causa intimer parece ser, no la distensión de la bolsa orávida ectópica, sino un derrumbe hormonal, una falta de luteína, que determina el desprendimiento de la cébil caduca uterina. Si esta producción hormonal se restablece a tiempo, y si el huevo no ha muerto aún, si supervivencia continúa, siendo ese ol caso de los embarazos ováricos llegados al término o cerca de él.

Los accidentes simpáticos del embarazo ectópico son tan marcodos como en el normal $y$ hasta pueden adquirir el carácter patológico; autores hay que citan casos de eclampsir.

Las reacciones biológicas son positivas, puesto que la inundación hormoral está presente aquí como en los eutópicos. Fesumiendo, los signos de probabilidad y de presunción son claros y si el huevo no muere o no es expulsado, se podrán tener los signos de certeza. 
Pero antes de dejar la primera etapa, es necesario anotar por su gran ocurrencia, dos cuadros agudos que se confunden en uno solo: el ictus hemorrágico y el ictus abdominal, indices ciertos de la muerte del huevo por ruptura del saco o por aborto. El ictus hemorrágico se manifiesta por la pérdida de sangre por la vagina, que es bien poca si se compara con ia que va quedando retenida en la cavidad abdomino-pelviana; la inundación se hace notoria en el fondo de saco de Douglas, que se vuelve tenso, renitente 0 crepitante y exquisitamente doloroso. Se ha constituído el hematocele pelviano.

A esto se agrega el ictus abdominal con un dolor agudo punzante localizado en el sitio de la ruptura, rebelde a los sedantes. Es, pues, un cuadro de abdomen agudo con gran dolor, gran defensa, facies peritoneal y ansiedad; lengua seca y saburral, hipotensión con pulso imperceptible, todo agravado por la anemia. Hé ahí el cuadro de un embarazo ectópico roto. de cualquier variedad. Los hay, sin embargo, que evolucionan sin mayores complicaciones y pueden llegar $\alpha$ término. Empero, lo excepcional y extraordinario es lograr la supervivencia riaterna y la fetal, como se leerá en la siguiente historia que nos suministró el doctor Jorge Solanilla, de Cali antiguo interno de los Servicios de Maternidad de Bogotá:

"R. A. L. 32. Cali. Negra vivandera y soltera.

Antecedentes generales: Fiebre tifoidea, malaria y R. A. A.

Antecedentes ginecológicos: Menarquia a los 13 años. Menorragias.
Antecedentes obstétricos Thes abortos de $2 \frac{1}{2}$ meses.

Anémica, desnutrida, paniculo adiposo poco desarrollado.

Corazón normal. Pulso 85. T. A. $110 \times 60$.

Aparato respiratorio, digestivo y urinario, normales.

Ultima regla: Agosto 24 de 1346.

Marzo 6: Altura uterina $25 \mathrm{cms}$

Pelvimetría externa: $191 / 2,201 / 2,241 / 2$, 2E $1 / 2$.

Feto único en situación transversa. Ruidos cardíacos fetales positivos, aunque velados. Diagnóstico: Embara$z \circ$ de 7 a $7 \frac{1}{2}$ meses con feto vivo.

Marzo 18: A. U. $28 \mathrm{cms}$. Feto único en situación longitudinal. Ausc. positiva. Embarazo de $71 / 2$ meses. Feto vivo. Fibroma uterino.

Marzo 31: A. U. $31 \mathrm{cms}$. Feto único en presentación de pelvis, que se moditica fácilmente. Auscultación posi. tiva.

La enferma desde su primera consulta se hospitalizó por estar edematizada. Salió el 5 de mayo y en la casa sintió dolores sacro lumbares y abdominales con intervalos de 1 hora io que la hizo regresar. A. U. no se precisa por la defensa abdomiral. Feto en situación oblicua. Auscultación positiva.

En los diferentes exámenes llamó siempre la atención la existencia de in tumor que hacía cuerpo con la "matriz", situado en su borde derecho. de consistencia firme y que fue tomado por fibroma. Además la palpación permitía apreciar und gran movilidad del feto y una gran depresibilidad de las paredes "uterinas". Se la pasa a la sala de partos para examen obstétrico completo. El tacto vaginal da el si- 
guiente resultado: Vagina amplia, profunda, de paredes congestivas. En la cúpula vaginal se aprecia una depresión de bordes mamelonados y blandos sin ningún orificio. En el fondo de saco derecho se aprecia una masa de consistencia firme, que se dirige hacia la fosa ilíaca del mismo lado y xi fondo de saco posterior. El fondo de soco anterior es muy profundo. No es posible tocar ningún polo fetal.

Como los datos de este examen son extraños y no fue posible apreciar el cuello del útero, se procede a un examen con espéculo, el cual demuestra la existencia de una vagina amplia, congestionada, con la misma depresión en su cúpula, ya apreciada por el tacto. Con esta exploración, tampoco the posible apreciar el cuello.

Entonces surge la ider de que se trate de un embarazo ectópico. Con el in de aclarar este diagnóstico, se inierroga a la paciente acerca de la rolución de su embarazo, y, a este sapecto, relata lo siguiente: días antes ce su primera falla menstrual comera sentir como "un peso" en la reón hipogástrica y al mismo tiempo norexia, estado nauseuso, dolor en la gión umbilical. A los dos neses de nenorrea aparecieron fuertes cólicos el bajo vientre, de una duración de os a tres horas, que se repetían cada mana. El dolor era pungitivo y se radiaba hacia el ombligo. Estos cócos venían acompañados de hemoragia vaginal de pequeña cantidad de un estado casi sincopal. Estas nanifestaciones dolorosas, se prolonzaron hasta el 25 de diciembre.

Ante este interesante caso y como las condiciones lo permitían, al siguiente día ( 8 de mayo) se hace un estudio radiológico, que muestra la existencia de un feto con las características de término, en situación oblicua y con la cabeza orientada hacia la fosa ilíaca izquierda y el polo pélvico hacia el hipocondrio derecho; la columna vertebral se encuentra fuertemente encurvada como si se trata1 a de un feto macerado. La pelvis se encuentra completamente vacía.

Intervencion: El día 9 de mayo, se ciecide intervenir. Se practican nuevos exámenes y se plantean otros diagnósticos como el de embarazo uterino con tumor previo.

Laparotomía mediana. Descubierta la cavidad abdominal, aparece un órgano muscular, muy vascularizado, surcado por gruesos troncos venosos Y de color, forma y consistencia como de un útero grávido normal. Se piensa en este momento, que el diagnóstico de embarazo extrauterino está errado. Se incinde el órgano como para cesárea clásica corporal, se extrae el feto, se aplica pitocín en el "miometrio", se introduce la mano en la cavidad para sacar la placenta; al practicar esta maniobra se nota inmediatamente que la cavidad tiene forma de un saco irrecular, con prolongamientos en forma de bolsas: la consistencia de la pared es firme en su parte anterior, $n$ así en la posterior. La placenta se encuentra adherida intimamente a las paredes. El saco no se retrae y comienza a sangrar abundartemente. Se extrae parcialmente la placenta; en este punto de la intervención se visualiza un órgano globuloso, de consistencia de músculo literino y que hace parte de la pared derecha del saco. La hemorragia aumenta considerablemente y se decide entonces practicar una 


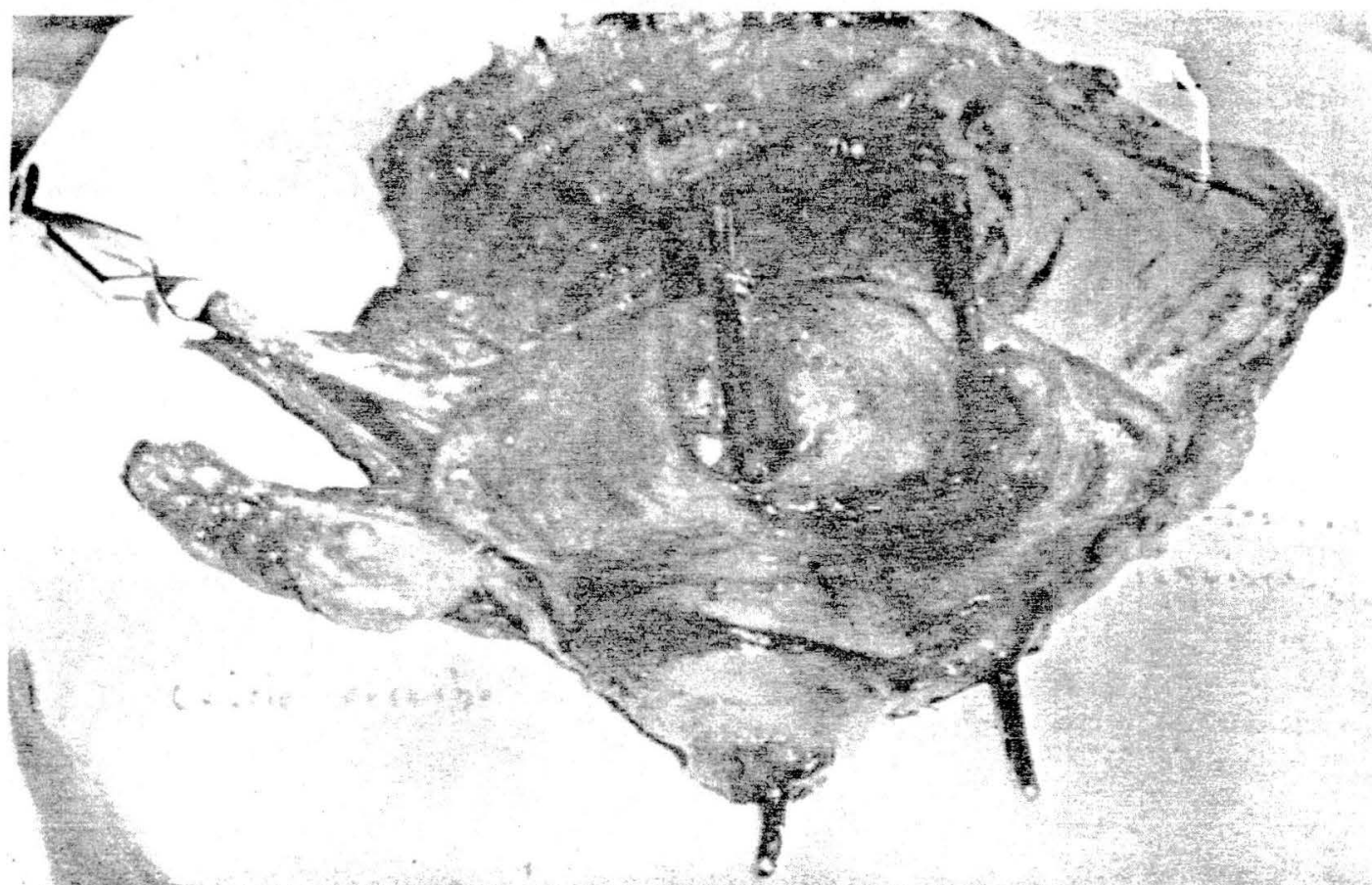

Pieza quirúrgica del caso relatado por el docior Solanilla.
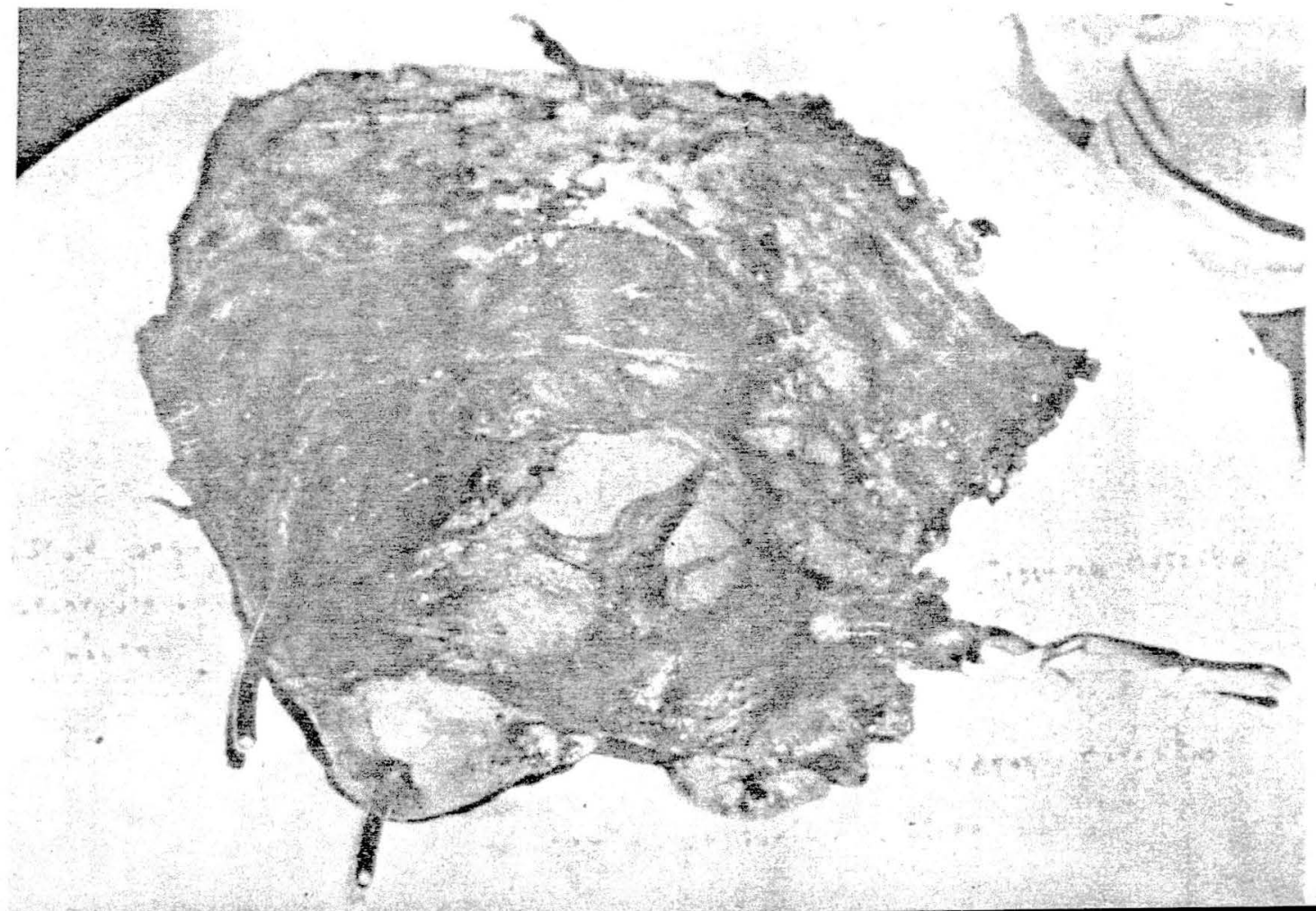
histerectomía total y extirpar el saco que se encuentra intimamente adherido al ciego, colon descendente, sigmoide y recto. La intervención se termina dejando unos drenes.

Tanto la madre como el niño sobrevivieron a la intervención.

En mi concepto trátase en el presente caso de un embarazo tubárico que se desarrolló a expensas de la porción ístmica de la trompa izquierda, gue permitió el desarrollo del embarazo mediante la hipertrofia de sus fibras musculares".

Hasta aquí es la fiel copia de la historia enviada por el doctor Jorge Solanilla, de Cali.

Otro caso bastante ilustrativo es el exominado y tratado por el doctor Pedro Londoño. Se trata de la nistoria 125.348 del servicio del Profesor Luque, en el Hospital de San Juan de Dios.

A. C. M. de 30 años de edad ha entrado al Hospital, con el diagnóstico previo de colecistitis.

Da como antecedentes importantes ser bien reglada, y haber padecido de thijo vaginal causado por una blenorragia. Su amenorrea data tal vez del 2.5 de septiembre de 1947. El flujo ya no existe. Tuvo un parto a término normal hoce 7 años.

Consulta por un dolor sordo, en veces agudo, pero siempre tolerable, localizado en la fosa ilíaca derecha con irradiaciones a la región sacrolumbar y cuya iniciación remonta $a$ $11 / 2$ mes atrás, sensación de peso en la pelvis y manifestaciones dolorosas en la región vesicular.

Los datos positivos del examen clínico son los siguiertes: Senos pigmeniados; tumor que ocupa la mitad derecha de la pelvis, no muy doloroso. de contornos poco nítidos y de eso movilidad; punto vearzular dolore El tacto vaginal indico: Cuello cc de orificio cerrado, blando, de pri para, rechazado hacia la izquierds Inirando hacia la derecha; cuerpo i rino aumentado de tamaño, biam rechazado hacia la izquierda y de 1 ma alargada; fondo de saco late derecho ocupado en su totalidad! una masa redonda, un poco doloro blanda, tija y nítida, separada de matriz, que ocupa el fondo de sc izquierdo. El tamaño de la masa es de una cabeza fetal y alcanza $\alpha$ or par también el fondo de saco pos rior. Reacción de Friedmann positi La radiología vesicular revela colec titis crónica.

El día 24 de enero es operada c el siguiente resultado, textualmer transcrito:

"Laparotomía media infraumibilia Abierta la cavidad peritoneal enco tramos una masa redonda, bland casi del tamaño de una cabeza fet surcada por enormes vasos sang: reos, que ocupa gran parte de la fo: lí́aca derecha y que corresponde un emborazo en plena evolución. O servamos ambién un cordón grue pálido, intacto en todo su trayecto que no es otra cosa que la trompa recha, la cual corre superficialme: la porción terminal del pabellón se cuentra involucrada en la masa n cionada. No fue posible identificc ovario correspondiente.

En la parte superior del fond saco de Douglas las asas intesti del delgado se amontonaban con parte inferior e izquierda del $t$ Observamos aquí la presencia $c$ queños coágulos sanguíneos 
tratar de extirparlos producen una débil hemorragia en la región superior derecha del tumor; encontramos el fondo del ciego invadido por las vellosidades placentarias, las cuales tamkién han alcanzado la parte ierminai del ileon.

Dicho tumor nos parece que ha crecido inicialmente entre las dos hojas ciel ligamento ancho para llegar a ocupar luégo la parte correspondiente a los vasos ilíacos externos. Es en este punto, donde encontramos lo que pudiéramos llamar su pedículo. En canLio, hacia la línea media y en la parte superior del fondo de saco de Douglas, las vellosidades placentarias han perforado la hoja posterior del ligamento ancho para llegar a la cavidad peritoneal e invadir el intestino delgado, formando lo que antes describinos como coágulos sanguíneos.

Procedemos a hacer una incisión en su parte superior, cerca de la trompa e interescando sólamente la hoja prarietal posterior del ligamento ancho. For divulsión logramos encontrar un flano que nos permite aislar el tumor y llegar con cuidado al sitio de implantación. Mediante sección entre pinzas hemostáticas logramos desprender las asas intestinales invadidas por el tejido placentario, teniendo el cuidado elemental de colocarlas un poco por fuera de su borde libre. Limitado el compo por compresas, ampliamos un poco más la brecha inicial y aprisionamos entre ciamps el cmplio pedículo placentario. Extirpado el tumor previa ligadura de la trompa derecha, ros queda el problema de los restos placentarios. Cortando de nuevo entre pinzas hemostáticas, logramos sacar gran parte fe ellos; sin que la extirpa- ción fuera completa, especialmente sobre el ciego y algunas asas de! delgado. Tales restos nos limitamos a tocarlos con tintura de yodo y peritonizamos la herida. No dejamas dren y cerramos la herida abdominal en cuatro planos, según la técnica clásica.

Post-operatorio normal.

El examen anatomopatológico microscópico, dice: Los cortes muestron trompas y ovario además de tejido placentario. En la trompa se aprecia hiperplasia de la capa muscular y in foco de tejido decidual; el ovario muestra relaciones de vecindad con la placenta. En esta última se aprecian vellosidades típicas, coágưios de sangre y áreas de necros's generalmente con formación de pus. Tanto en el ovario como en la trompa hay también reacción inflamatoria. Diagnóstico microscópico: Embarazo tubo-ovárico.

Se extrajo un feto masculino nor$\mathrm{mal}$ de $17 \mathrm{cms}$. de longitud, cordón umbilical de $20 \mathrm{cms}$. de largo, placenta íntimamente adherida a la trompa y al ovario".

El doctor Rafael Ramírez Merchán. publicó en la Revista MEDICINA y CIRUGIA, de junio de 1946, otra historia que podría colocarse en este capítulo y que por lo ya conocida, no la relatamos textuaimente.

Se trata de una primípara de 25 años que aunque ignora la fecha en ơue sobrevino su última menstruación, asegura que empieza a declinar en el g? mes de su gestación. Todos sus aparatos de la economía son normales y a primera vista, dice el articulista, "un ambarazo normal casi a término es io que merece la atención".

Los datos obtenidos por el interno dan por resultado el diagnóstico de 
un embarazo de $81 / 2$ meses (altura uterina $30 \mathrm{cms}$.), con feto muerto pues "no se aprecion con claridad partes fetales y la auscultación es negativa"; el parto no se anuncia todavía ya que a' tacto vaginal practicado se apreciata "cuello largo, cerrado, presentación móvil por sobre el estrecho superior".

Sometida a observación, en espera ciel desencadenamiento del trabajo, queda en el servicio por 20 días, sin oue se obtenga el parto y luégo se pone en práctica el procedimiento de Watson con un fracaso rotundo. Corridos ya 50 días de permanencia en el Hospital, los médicos tratantes quisieron resolver el problema de este embarazo anómalo por la vía baio, pèro les fue imposible pcrque la esclerosis y la dureza del cuello uterino impedian su dilatación.

Restaba sólo una alternativa y era ia intervención quirúrgica por vía abdominal; en electo se practicó y cuando se creyó poder realizar una operación cesárea para extraer el feto rnuerto, se tuvo una serie de sorpresas.

Hecha la laparotomía los cirujanos encuentran algunas adherencias epiploicas pero no les conceder importancia; ya sobre el órgano gráyido se ve un peritoneo visceral "vascularizcdo en forma extraña y que lo que debía ser el segmento inferior dificulta un poco el aislamiento de su peritoneo porque tiene tal contextura el dicho órgano, que en seguida va a ser d:fícil su disociación".

Extraído un teto a término muerto $y$ macerado, de 2.200 gramos, se hacen tracciones sobre el cordón para extraer lo placenta, y se nota que la bolsa grávida se viene con los anexos y no sangra ni siquiera después de que se arrancan trozos degenerados de placenta. Ante este cuadró se pensó en extirpar el órgano degenerado, llevando las cirujanos la idea de una histerectomía. Para lograria aislan bien la bolsa, liberándola de las "pocas adherencias del epiplón y de otras tántas de algunas asas intestinales" y encuentran con sorpresa que en la parte más inferior y en su cara posterior hay un órgono esculpido que no es otro que una matriz "aparentemente sana y no grávida".

"De consiguiente, dice el, doctor Ramírez, A. R. C. nos proporcionó la rareza de un embarazo ectópico casi a término".

\section{II - Embarazo Ovárico.}

Generalidades: Al paso que la gestación tubárica es un hecho indiseutible y de frecuente observacion, no sucede lo mismo con los embarazos ováricos y abdominales.

Multitud de razones se aducen para negar la probabilidad de que el óvulo. una vez fecundado, comience sus transformaciones, emita sus vellosidades coriales y se fije ya sea en el ovario o en la cavidad abdominal. El de:precio de los tratadistas hacia estas dos entidades gineco-obstétricas inthye el ánimo en el sentido de que sólo rarezas son estus dos implantaciones eftrouterinas del huevo fecundado.

Para quienes aceptan el embarazo ovárico, se puede hacer una división en superficial o profundo, según que el espermatozoide haya alcanzado a la célula hembra, después o antes de la ruptura del foliculo moturo. 
Etiologix El factor edad no es deierminante, pero se observa que la mayoría se presenta entre los 20 y ios 35 años.

Recassens y otros afirman que es r.ás frecuente en las multíparas, mientras otros manifiestan que es mayor si? incidencia en las primigestas. En opoyo de estos últimos está el factor infecundidad relativa, ya que muchas rnujeres que acuden al médico portando una ecciesis de cualquier tipo, can el antecedente de una infecundidad más o menos prolongada. Davis se expreșa así: " $\AA$ la esterilidad previa no se le ha dado el suficiente énfasis como factor etiológico, pero la esterilicad relativa parece ser un factor real. Shuman dice que el $30 \%$ de las mujeres que han tenido un ectópico, llevan consigo una historia de esterilidad".

Una hipótesis muy sugestiva para explicar la implantación extrauterina, es el rápido proceso de desarrollo del huevo y su procoz capacidad de fijación. En el caso contrario, en que la capacidad fijadora fuere demorada, el huevo pasaría de largo por toda la superficie uterina para verir a implantarse en la porción segmentaria (placenta previa), en el cuellio o aun $\equiv n$ la vagina.

Anatomía Patoiógica: La comprobación de una ecciesis ovárica es bastar.te difícil, por cuanto las adherencias a los órganos vecinos, trompa y matriz se hacen prontamente y porque después del crecimiento no se puede ciecidir si la situación del tejido placentario es primitiva o secundaria.

Para solucionar este problema Spiegeiberg estableció cuatro bases que de ser positivas dan el diagnóstico cierto de embarazo ovárico; son estas: i) La trompa del lado afectado debe estar intacta; 2) El saco fetal debe oc.. par la situación del ovario; 3) El saco ovárico debe estar conectado con el úiero por el ligamento útero ovárico, y 4) Se debe hallar tejido claramente ovárico en la pared del saco. Llenados estos requisitos estará establecido el diagnóstico.

Ahora bien, el desarrollo del huevo puede hacerse en el interior del iolículo o en la superficie externa del ovario. Si ha sucedido lo primero, se pọdrón ver células luteínicas del cuerpo amarillo alrededor del saco; las modificaciones deciduales tienen lugar en todo el tejido conjuntivo de vecindad y hay hiperplasia e hipertrofia de las células luteínicas de la teca inte:ria; las vellosidades coriales penetran en el parénquima y la placenta se desarrolla en el interior del ovario. Si la implantación ha sido superficial, la placenta se fijará no sólo en el tejido cvárico, sino que también se dirigirá hacia la trompa para hacerse más amplia; el saco fetal crecerá hacia la cavidad abdominal.

En cuanto a la duración del saco ovular y luégo fetal, se puede decir que tiene grandes posibilidades pora llegar a término, pues que los tejidos propios son más susceptibles que los de las trompas para suministrar unas paredes resistentes de protección. Siantiti, en uno de sus 37 casos observó que el ovario estaba transformado en una pared quística de 1 a 3 milímetros de espesor y Schorsch, publica un embarazo ovárico a término con fete vivo. Entre nosotros son elocuentes los casos publicados por el Prolesor Parifirez Merchán. 
En cuanto a los ováricos superficiales, en los cuales el óvulo se ha atascado en una de las muchas hendiduras del epitelio ovárico, es de aceptación común hoy día, que ello no se realiza al acaso, sino que debe haber para que sea posible, un islote endo. metriósico en la superficie ovárica. $Y$ es claro, porque el glicógeno, necesicad primera del huevo, sólo se halla en las células deciducles y éstas son patrimonio del corion de la mucosa uterina. Y si a esto se agrega la capacidad de reacción decidual del tejido ovárico, se puede esperar un buen crecimiento el huevo fecundado. aunque con menos posibilidades de cvanzar en la variedad profunda.

Curso Clínico: Es muy difícil es:ablecer diferencias clínicas entre un embarazo ovárico y otro abdominal. Por esta razón, aunque el título de este capítulo es el de embarazo ovárico. incluiremos en él lo que podríamos decir de la evolución del embarazo abdominal. Asímismo, dejaremos de lado los signos del principio, ya que se confunden con los del común embarazo tubario, descritos unteriormente.

Claramente se aprecian las parte: fetales y los movimientos, así como los ruídos cardíacos del feto, con una ritidez absoluta debido a la presencia de una pared menos gruesa; las sensaciones subjetivas y los signos de comprensión son también más intensos.

Las metrorragias pueden acompañar caprichosamente el curso de la gestación o estar ausente.

Llegado el término del embarazo, sobreviene el "falso trabajo", con contracciones que dilatan el cuello hasta cierto punto, provocan la expulsib. del tapón mucoso y obligan los preparativos para recibir el nuevo sér. Fero este falso trabajo da valor a su nombre, desapareciendo por completo de un momento a otro, dejoncio como saldo la desaparición de los movimientos fetales y de los ruídos cardíacos. Inícianse luégo las transiormaciones post-mortem del feto retenido.

Si la eáad no es avanzada, pueden los tejidos resolverse lentamente, pero si esto no sucede, otras transformacines han de verificarse para que el organismo materno se defienda del cuerpo extraño o lo efimine fuera de sí.

Lo más frecuente es la infección del quiste fetal, favorecida por las adherencias a las vísceras contiguas. Ese quiste infectado se fragua su camino hacia el exterior, perforando una de las vísceras huecas o yendo hasta la pared abdominal para fistulizarse. Ia salida del esqueleto fetal y de ios tejidos infectados de la bolsa gróvida se realiza progresivamente y aparecen por la vagina, uretra, recto o a traves de la pared abdominal.

Otra transformación posible, la segunda en frecuencia, y la más favorable para la mujer, es esta: Se verifica la absorción de todos los líquidos de! feto, quedando una masa macerada. Luégo se depositan las sales calcáreas y el esqueleto adquiere la consistencia de piedra, quedando asi constituído el litopedion. Pero esto puede hacerse no ya en el cuerpo fetal, sino en sus membranas de envoltura y en la placenta y entonces se tiene el litokelifos, o finalmente pueden ounarse las petrificaciones fetal y anexial, dando lugar al litokelifospedion. 
El tiempo necesario para que tenga lugar la estructuración de estos cambios es considerable, pues el proceso es lento y se asiste a la reabsorción del liquido amniótico, a la momificación de las partes blandas y luégo si al depósito de las sales calcóreus. En todo caso, es cuestión de años.

Esa masa pétrea y disminuída de rolumen puede ser llevada por la mujer durante mucho tiempo, sin que produzca molestias graves y aún más puede ser muda en sus manifestaciones. En la literatura mundial se citan muchos casos de litopediones, pero de litokelifospediones sólo hemos encontrado uno, el de Penick, de Oklahoma, después de revisar el Index Medicus de 1943 a 1946. Como hecto raro, podemos traer a cuento lo observado por Virchow, citado por Recassens, de la conservación de la estructura normal de algunas partes blandas del quiste fetal retenido, debido a la persistencia de la irrigación sanguínea.

Formado el litopedion, terminan los procesos defensivos de la mujer y en la mayoría de los casos no es obs. táculo para que continúe la vida gerética de la paciente, salvo en algurios casos en que puede constituír una distocia por tumor previo.

Diagnóstico: En páginas anteriores cuedó esbozado el cuadro clínico de un embarazo ovárico o abdominal a través de toda su evolución; encontrado ese conjunto de datos anamné. sicos lejanos (menarquia taraía, esterilidad relativa, posibles infecciones anexicies) y hallados los signos propios de esa ecciesis, necesitamos comprobarlo por otros métodos.
Las reacciones hormonales siempre son positivas, pero dan un resultado más débil que en los embarazos nor. males; esto es particularmente para la prueba de Ascheim y Zondek, siendo más segura en cambio la de Friedman.

De un menor valor es la reacción de sedimentación de Fahreus, pero útil cuando en los primeros tiempos de la evolución se alberga la duda de si realmente, es un ectópico o un proceso anexisalpingítico o apendicular. Ligeramente aumentada, entre 25 y 35 a la hora, puede inducimos en.ta. vor del primero, pero un aumento ma. yor estará en relación con los procesos infecciosos.

Otro procedimiento diagnóstico patrocinado por Klaften es aquel, mediante el cual, por iluminación pélvica a través de la vagina puede verse un halo rojizo más o menos intenso alrededor del sitio de hemorragia, ruando ha habido ruptura del saco.

Además de esta diafanoscopia $y$ para el mismo caso de la ruptura del embarazo ovárico, es de gran valor la punción exploradora del tondo de saco de Douglas, pues el encuentro a use nivel del contenido hemático, casi con seguridad nos da el diagnóstico le ectópico roto, con la sola saivedad quizás, y muy rara, de la ruptura del pedículo de un quiste torcido del crario.

En cualquiera etapa evolutiva del embarazo ectópico, pero particularmente en los primeros meses, cyuda mucho el nuevo método de la peritoneoscopia.

Carlos Heuser, en 1921, introdujo la histerosalpingografía como método diagnóstico de las ecciesis y posterior. mente otros autores han hecho pubi:- 
caciones sobre sus experiencias en casos de emborazos abdominales, con resultados favorables. Supera a la simple radiografía porque ésta no sefiala sitio donde se asienta el esqueleto fetal y pasan inadvertidos embarazos que se inician.

Historias Cínicas: Cito en primer lugar las publicadas por el docior Ramírez Merchán, por ser las más interesantes.

Es la primera la correspondiente a una secundípara a término, con 35 cños de edad, que tuvo su última regía el 5 de junio de 1942 y dió a luz a su primer hijo hace 6 años.

Esta señora, contestando el interrocatorio asegura su estado grávido y añade que tuvo sensación de movirientos fetales has un mes y medio chtes, época en que sufrió un fuerte trcumatismo abdominal; de entonces para acá ha visto con sorpresa que su abdomen, lejos de continuar su crecimiento, ha ido disminuyendo.

La altura uterina es de 26 centímetros, las partes fetales no se perciben claramente, la auscultaciór letal es negativa y el estado en que ce halla a cuello es largo, cerrodo y de consislencia dura.

Después de una radiografia en que se aprecia el esqueleto de un leto muerto, se procede a estimular las contracciones uterinas con el método de Watson, y visto su fracaso, se obtiene otro al pretender dilatar al concucto cervical.

La intervención quirúrgica por vía clita, fredionte la cual se extrajo el saco fetal y el feto muerto y macerado, de 2300 gramos de peso, dejó claramente establecido que la matriz, indepen- diente de la bolsa grávida, esta integra.

Enviada la pieza quirúrgica al Laboratorio de Anatomía Patológica el áoctor Sánchez Herrera da su corconcepto en los siguientes términos: "Diagnóstico microscópico: Embarazo probablemente de ovario. Leiamioma múltiple. El material examinado muestro placenta necrosada, cordón umbilical, peritoneo, coágulios de sangre Y tragmentos de tejido que, de manera muy imperfecta recuerdan ovario. Las vellosidades placentarias no presentan sino elementos celulares en estado de desintegración. En el cordón umbilical hay necrosis. En el peritoneo se ver áreas de hemorragia. dilatación de los vasos lintáticos hiperplasia del tejido conjuntivo $e \mathrm{in}$ filtración leucocitaria poli y mononuclear. El tejido que recuerda el ovario forma parte de la pared secular. Los cortes practicados muestran un neoplasma benigno, formado por tejido muscular liso y tejicio conjuntivo fibroso con áreas extensas de necrosis. Es encapsulado y en la periferia se aprecia bien la estructura".

Más interesante que la historia anterior es la siguiente, en la cual se hizo el diagnóstico clínico, confirmado luégo plenamente

Se trata de una paciente de 42 años de edad, casada y con vida genital activa. Antecedentes ginecológicos: Menarquia tardía a los 17 años, menometrorragias y flujos vaginales amarillentos, hace 2 años.

Antecedentes obstétricos: Un parto normal a término hace 21 años y otro prematuro de 7 meses, al ono siguiente. Infecundidad de 20 años. 
El embarczo actual tuvo su comier:zo por el mes de julio de 1945 , manifestóndose con todos los signos subjetivos y objetivos propios del estado crávido. Su curso más o menos norrnal, se vió alterado al go mes, por la cesación de los movimientos fetales, una pequeña pérdida sanguínea vauinal y la aparición de una calostrorrea abundante.

Dos meses después de terminado $\in l$ límite normal de su preñez, acude al hospital y en un primer examen practicado por el Interno, se llegó al diagnóstico de un embarazo típico a término, con feto muerto. Posteriormente e! doctor Ramírez Merchán, a la sazón Jefe de Clínica, practica cuidadosamente examen tocoginecológico a la señora, dándose perfecta cuenta de que la tumefacción abdominal de 28 centímetros de altura, no correspondía a la 1.atriz, pues sus movimientos no ercn sclidarios con los del cuello uterino $\mathrm{y}$ caquélla podía precisarse aunque no muy claramente.

Basado en estos datos y ayudado por una anamnesis verídica y en los signos de probabilidad del embarazo, plunteó el diagnostico de un embaiszo ovórico a témino con feto muezio. Y luégo retorzándolo con otros hechos que concurrion de coniunto, hizo más fundado su acerto; éstos eran:

a) Menarquia tardía a los 17 años.

a) Infecundidad de 20 años.

c) Antecedentes de flujo vaginal (ndice de infección).

d) La edad de la paciente, factores que se invocan como etiológicos del embarazo ectépico y estos otros propios de su evolución: a) Amenorrea de 11 meses.

b) Faiso trabajo al $9^{\circ}$ mes.

c) Metrorragia concomitante.

d) Calostrorrea espontánea concomi. tante.

e) Supresión de los movimientos fotales después del falso trabajo.

f) Disminución de la tumefacción ab. clominal.

g) Crisis de irritabilidad simpática

h.) Independencia entre el tumor aodominal y la matriz

i) Examen radiológico del abdomen e histerosalpingografía que por ser ds gran importancia copio textualmente:

"En la radiografía simple encontramos un embarazo que presenta todos los signos radiológicos de feto muerto tales como el de Horner Spalding, exaceración do la curva de la columno vertebral y el signo de Mathews, los cuiales todos son positivos. También aparece una alteración del líquido cmniótico porque encontramos una densidad más elevada que la normal". Anotaba además verbalmente el radić. logo que las paredes del continente eran densas y que su situación mediana, así como el desarrollo fetal, hacian pensar que quizá el continente ro podía ser otro que la matriz. Ferc ante la insistencia nuestra se practicb́ la histerosalpingografía en la cual encontramos que la matriz se inyecta normalmente y su tamaño y forma son normales, pero su situación ligeramento desviada hacia la derecha. Aumentando un poco la entrada de Yodatol y por consiguiente la presión endoute. rina se loora hacer pasar la substancia opaca a las trompas; "tanto del lado derecho como del lado izquierdo se inyectan casi en su totalidad". 
Intervenida la señora el 26 de junio. $\varepsilon e$ encontró en su rvidad abciominal un tumor con adherencias epiplóicas e intestinales que cuidadosamente se liberaron y se extrajo el quiste $\sin$ abrirlo y luégo de ligar su pecículo

"Al revisar los órganos genitales internos se encuentra la matriz del tonaño normal. Los anexos del lado derecho quedaron íntegros y sanos; los cel lado izquierdo formaban cuerpo con el tumor que se extirpó".

Al abrir el tumor se vieron sus paredes musculares y fibrosas y salió de el gran cantidad de líquido amniótico de color verde y con meconio y un feto bien formado de 1.400 gramos de peso, unido por el cordón a una placenta delgada que adhería íntimamente a las paredes del quiste.

La descripción histológica es la siguiente: "Embarazo tubo-ovariano. los cories muestran ovario y tubo uterino. Además se encontró placenta err estado de desintegración situada aparentemente entre sl tubo y el ovario. Tanto el ovario como el tubo, pero especialmente en este último, se ve reacción inflamatoria muderada con infiltración leucocitaria poli y mononuclear. Edema".

\section{El doctor Nelson Restrepo, en sid} Tesis de Grado, en que trata los errores diagnćsticos en Ginecología, relala el caso de una señora de 48 años que ha dado a luz a 5 hijos y a un aborto de tres meses hace 5 años. Padeció de flujo vaginal amarillo en diciembre de 1947 y su última regla vino el 20 de enero de 1948. Se hospitalizó por dolores en la fosa ilíaca :zquierda, intermitentes y de tipo cólico; también tiene dolores en el hombro derecho. El examen general es satis- taciorio y el ginecológico suministro los siguientes datos: Vagina normal. Los fondos de saco menos el anterior se encuentran abombados y no dolorosos. Cuello dirigido hacia atrás, cer:tral, móvil y de orificio transversal cerrado. Se toca una gran masa que ccupa toda la pelvis, abomba en iodos los fondos de saco, es dura, no dolorosa, poco móvil, regular y está en relación íntina con la matriz de la cual no se puede delimitar.

Con el diagnóstico de Leiomioma uterino se intervino, encontrando una gran masa regular, blanda que scupa toda la pelvis y que da la impresión de pertenecer al útero, firmemente adherida al asa sigmoide. Se libera el sigmode, se continúa por el lado derecho, $y$ se encuentran la trompa y el ovario de ese lado. Por detrás de la trompa se encuentrr un plano de desprencimiento por donde se empieza a separar al tumor. Se pasa después al iado izauierdo y se sigue la liberación. del tumor, separando su cara anterior de la posterior del cuerpo del útero. u la que se halla fuertemente adheririo. Liberación iotal. Trompa y ovario derechos sanos. Trompa y ovario izcuierdos destruídos totalmente. Exti:pación del tumor.

Diagnóstico Quirúrgıco: Embarazo uvárico con leto muerto, macerado de unos 25 cms de longitud.

El doctor Francisco Afanador Jiménez operó el 24 de octubre a una serora que había sido tratada como para una amenaza de aborto, pero que al examen clínico cuidadoso mostró tener un embarazo ectópico, ial 
vez ruto L. hallazgo quirúrgico consistió en una gran masa del tamaño de una cabeza fetal, localizada en el ovario derecho y cubierta totalmente por epiplón. Al desprender las adherencias a la trompa homónima se rompe súbitamente la masa dejando 'escapar gran cantidad de líquido citrino y poniendo así al descubierto un feto vivo de $14 \mathrm{cms}$. de longitud que dejó de respirar pocos momentos después La placenta estaba insertada en el cvario, el clego y la terminación del intestino delgodo.

Diagnóstico quirúrgico: Embarazo ovárico.

\section{III - Embarazo Abdominal.}

Generalidades: En cuanto al embaraco abolominal, aun los escépticos crepton, con quienes lo afirman, que puede ser secundario o primitivo; en el primer caso sería consecutivo a la ruptura o a un aborto tubario, mientras que en el segundo se trataría, omo su nombre lo indica, de la imiantación y crecimiento del huevo en eno cavided abdominal.

Esta última es la variedad le gescción más combatida y con menos vieptos.

Williams parece ser el más enfático a negar el embarazo abdominal priritivo, cuando en una parte de sil iratado se expresa en el sentido de ue todo embarazo considerado como al se debe a un aborto tubario. Bland Sutton va más lejos cuando niega do lano la posibilidad de su presencia on la mujer y en los animales inferioes. Pero el mismo Wha ams cita a First y a Knipe y a Waller y jacquin.
- wienes, "sin embargo, han sisscrito ejemplares que casi llenan los requisitos exigidos". Más adelante acoge como tal, uno de seis semanas inplaritado en el mesenterio de la llexura sigmoidea "que parece estar fuera de toda crítica", descrito por Reifferachaid. William E. Studiford publica en el An. I. Obst and Gin. (Vol. 144, pág. 487), de 1942, una historia de preñez (bodominal primitiva y que posteriormente vimos citada en el texto de Novak.

En resumen y ateniéndonos a las opiniones de los tratadistas, si son posibles, han existido, y seguirán presentándose embarazos abdominales primitivos, aunque con carácter de rareza relativa, lo que les impriine un aspecto espectacular y trascendente.

Etiología: Fuera de los factores etiológicos estudiados a propósito diel emharazo ovárico y que son comunes a lo variedad abdominal hay estas otras causas.

Las trasmigraciones o supermigraciones internas saltan a la vista como causas probables del embarazo abdorinal primitivo y, con mayor razón si el óvulo en su carrera encuentra este otro factor de la endometriosis ovárica o peritoneal, al cual los cutores modernos conceden una gran importancia. P. Vara opina que la implantación del huevo en estos islotes encometriósicos intraperitoneales, no puede ser nunca la mera casualidad y junto con muchos autores cree que se trate de un tactismo positivo del huevo hacia la parcela onormal de encometrio. Frankel y Schenk trabajando sobre 29 casos de embarazo ectópico, pudieron concluír que el factor endometriósico ero esencial. 
Y por otro lado Víctor Conill, no cuita toda respoi.sabilidad al espermatozoide y dice: "Quedamos en libertad de pensar cue el espermatozoo, elemento activo, puede escoger, comio un vertebrado superior, el terreno más próximo y propicio a sus operaciones vitales"

Como ctras causas posibles estarian las maliormaciones congenitas, los tumores pelvionos, los quistes del ovario, etc., es decir, toda lesión que deforme 0 alargue el trayecto que sigue el óvulo o también, como dice Schoenholtz, podría tratarse del fracaso de la aspiración tubaria.

Anatomia patológica: Aquí como en el embarazo ovárico los autores modernos Sampson, Schenky y Frankel, están de acuerdo en que su producción sólo es posible con la preexistenwa de una zona endometriósica transrortcda a la serosa peritoneal o a un orgaro abdominal por movimientos antiperistáliticos de las trompas durante la descamación menstrual, o como lo sostiene Recklinhausen, porque estén allí depositados como restos embrionarios mullerianos, o aún que hayan sido formados por metaplasias de la serosa como lo afiman las teorías de Ivonotf.

Una vez caído el óvulo a la cavidad peritoreal y fecundado por el espermatozoide, busca su sitio de implantación ya en el fondo de saco de Douglas, ya sobre lq cara anterior del recto, ora en la posterior de la matriz - por último en la cara externa del ligamento úterosacro. Pero también se puede hallar en el mesosigmoide o in pleno epiplón.

Posteriormente y a medida que avanza el crecimiento, la placenta puede fijarse en todos los órgonos intraperitoneales que encuentre $a$ su alcance, fijándose de esa manera en ol intestiro, la vejiga, epiplón, etc.

"Para establecer el diagnóstico de un embarazo abdominal primitivo, es condición básica que el aparato genital aparezca absolutamente normal".

Las membranas de envoltura estarím. engrosadas por otras de neotormación a expensas de la serosa.

Tanto el embarazo abdominal primitivo como el ovárico han constituído su capítulo anatomo-patológico a base de hallazgos laparotómicos.

Las modificaciones histológicas de los órganos genitaies son las mismas cue en el embarazo tubario y por esramos los cambios uterinos, tubarios, vaginales, mamarios, así como las modificaciones generales del organismo femenino.

Historias: Caben aquí las historias presentadas por el doctor Roberto Serpa, como Trabajo de Agregación, en las cuales no se sabe ciertamente el diagnóstico de implantación, pero que interesan por cuanto han tenido un tar plenamente estudiados no enumedesarrollo abdominal y por las transformaciones sufridas por los fetos retenidos.

En primer lugar relata el caso de ina señora de 37 años que habia tenido 5 partos normales y "un aborto" cinco meses antes de ser vista por el doctor Serpa.

Los signos de ese aborto tueron fuertes dolores en el bajo vientre y en las caderas, acompañados de hemorragia uterina y de la expulsión de una bolsa que resolvieron bautizar como embrión. Pasó dos meses en relativa mejoría pero cualquier día tuvo 
nuevos dolores abdominaies que fucron achacados esta vez, a una hernia estrangulada.

Al llegar el doctor Serpa a la casa de la enferma se encuentra con un cuadro de abdomen agudo: Facies peritoneal, voz débil, gran dolor abdorrinal, defensa de la pared, lengua sahurral, pulso pequeño rápido e intermitente, $371 / 2$ grados de temperatura axilar, gron constipación.

Bajo la acción sedante de una ampolleta de sedol, pudo palparse un tumor abdominal de consistencia heterogénea e irregular en su forma, que dolía a pesar de la droga. "En ia zona umbilical y en los flancos daba la blandura de un quiste del ovario y en os hipogastrios se hallaba dureza de homa y en la tosa ilíaca izquierda se alpaba un cuerpo esférico y duro omo un coco".

Los tactos vaginal y rectal enseñaon al doctor Serpa que la masa abominal era independiente de la ma$z$ y lo hicieron pensar en un embazo ectópico, impresión que se conmó al percibir movimiento del tumor. Decidida la intervención y luégo de a junto médica, fue operada la enma en nuy malas condiciones or. micas. E! resuitado tue: "un feto enelto en sus membranas entre una tidad de liouido amniótico normal; cabeza ocupaba el pezueño banete $y^{\prime}$ el resto de cuerpo ascendía n el abdomen".

Al sacar el niño uno de sus miemros desgarró la placenta; ésta más rande que lo norma!, se insertoba en estómago, en el colon transverso. n el epiplón mayor y en muchas asas utestinales; su desprendimiento labooso ocasionó copiosísimas y extensas hemorragias que fueron cohibidas convinientemente:

Las condiciones en que se había realizcido la operación, sumadas a la pérdida sanguínea y al choque peritoneal, llevaron a la muerte a la señora $M$. de $R$. tres días después, al paso que el feto vivo de 3.500 gramos siguió su desarrollo normal.

En su segunda historia el doctor Serpa presenta otro caso muy interesante. Es el siguiente:

A. G., señora de 30 años y madre de 7 hijos, llega al Hospital de San Juan de Dios el día 10 de abril de 1915.

Hay un antecedente de importancia en ella, el cual es una blenorragia.

La enfermedad actual data de tres años $y$ evolucionó en los primeros meses como un embarazo normal, y al cumplirse ellos, se le presentó un talso trabajo: la tumefacción abdominal siguió con los mismos caracteres desde ese día hasta cuando ingresó al rospital; padece un dolor continus oue se exagera al caminar o al tocorse, en la fosa ilíaca derecha, en $A$ flanco derecho y en la zona umbilical. For palpación se observa un cuerpo de consistencia dura y que se descioin ligeramente al imprimirle movimientos; localizado en el flonco derecho y er. la parte superior de la zona umbilical; $\in n$ el resto de esa zona $y$ más hacia la derecha hay cuerpos mas pecueños e igualmente duros. El examen vaginal permite darse cuenta de que dichas masas no hacen cuerpo en la inatriz, que está alojada en $\in$ l lado contrerio.

Con estos datos hizo el doctor Serpa el dicgnóstico de embarazo extrauterino litopedión, y se vió apoyado pcr e! del Profesor Rafael Ucrós. 


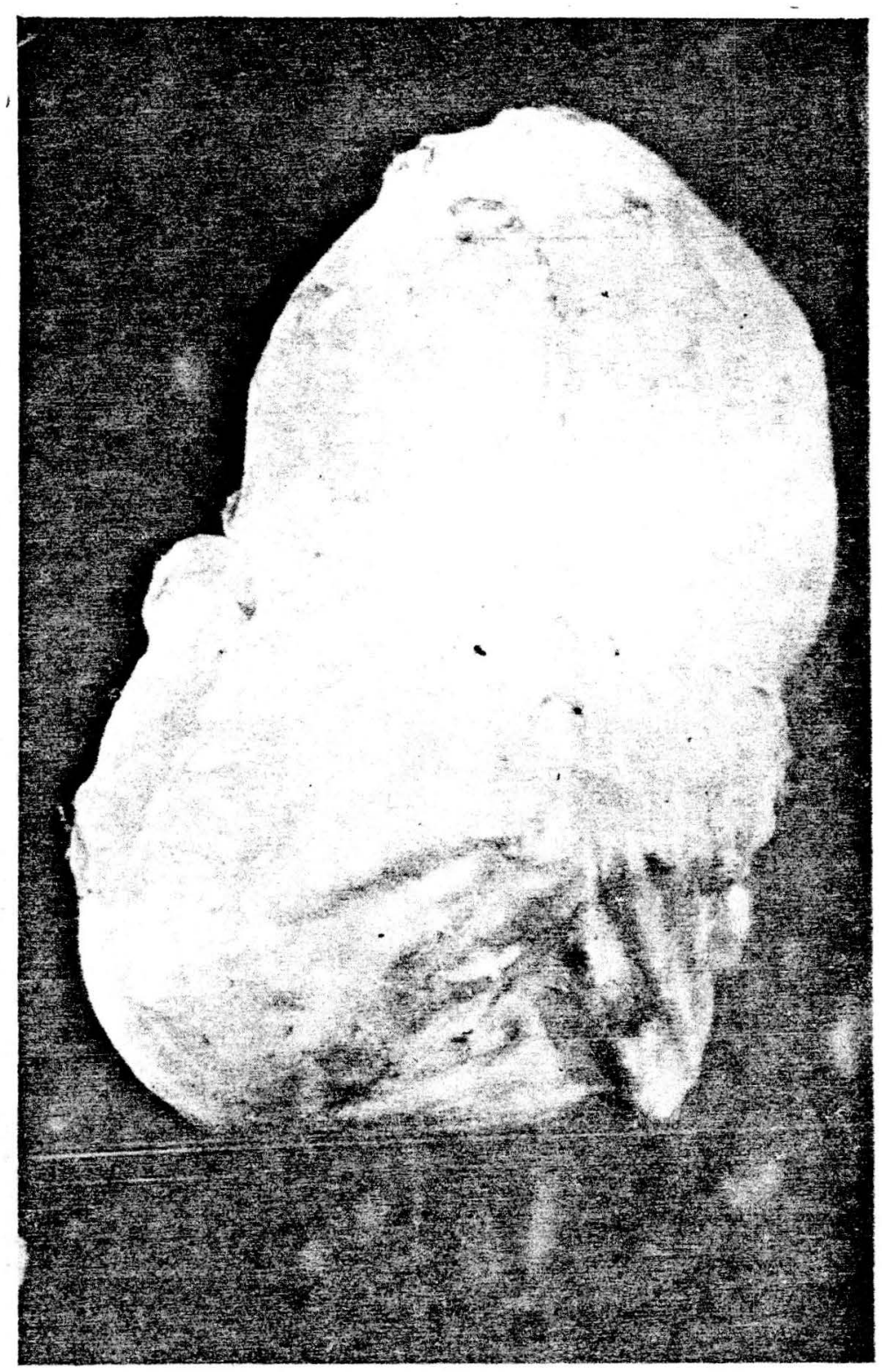

LITOKELIFOSPEDION 
El relato de la intervención es textualmente el siquiente: "Narcosis clorofórmica. Laparotomía media. Intraumbilical. Abierta la cavidad abdominal se apercibió cubierta por el gran epiplón, un cuerpo de color blanco racarado, prendido por un pedículo ćseo a un cuerpo ovoide que, a su vez, estaba ligado a la trompa izquier. da y adherido al recto y a la losa ilíaca. Siendo imposible la diseccior. del epiplón, se cortó después de haber ligado sus vasos. Se desprendieron los cuerpos, que no eran otra cosa que el feto y su placenta, de las adherencias al intestino y al recto y cortada la trompa se extrajo el litopedion y su placenta. El ovario derecho tenía un quiste sanguíneo al que se le hizo la inipuntura".

Otro caso interesante es el de la seFiora I. S., de 38 años de edad, modre de cinco hijos; fuera de ellos tuvo un embarazo el último, que transcurrió con algunos dolores a los cuales no jes concedió ninguna importancia.

A los nueve meses, sobrevino un falso trabajo y luégo la sospecina de un maleficio por la desaparicion del hijo.

Cinco o seis semanas de relativa tranquilidad y luégo se presentan dolores abdominales, calorío, apertura de la tumoración dolorosa a nivel del ombligo y salida de gran cantidad de pus Falpando el abdomen se percibía und crepitación ósea neta y se favorecia la expulsión del pus delgado y moreno. Estaba hecho el diagnóstico de feto macerado e infectado con la apertura ciel quiste a la pared del abdomen.

La necedad de la enferma no permitió ninguna intervención. Días después se asistió a la expulsión por el crificio fistula de una clavicula y luégo un fémur y posteriormente otros huesos que la misma enferma extraía, con la esperanza de que al salir todos los podría enterrar en tierra santa.

$Y$ en su última historia presenta el Protesor Serpa, a N. N., mujer de 40 cinos, quien llegó al Hospital de Bucaramanga en diciembre de 1947, para consultar por un tumor abdominal.

Seis años antes había tenido su sexto embarazo, el cual llegado a término, páreció concluír cuando en realidad sólo hubo un falso trabajo $y$ luego la cesación de los movimientos fetales y el decrecimiento del vientre.

Por palpación abdominal se encuentran los miembros duros e inmóviles de un feto litopedizado, cuyo polo cefálico estaba en la fosa ilíaca izquierda.

Operada la enferma se halló un feto calciticado, unido a una placenta ovoidea y negruzca y libres ambos de toda adherencia; sólo una parte del epiplón cobijaba y adhería a una extensión del teto.

Finaimente relatamos el caso interesanie que ha motivado este artículo. Advertimos que la historia ro es tan detallada como los demás, pues la pacjente estuvo durante mucho tiempo ausente del consultorio de su médico de tamilia, y además, porque $e$ diagnóstico previo fue solamente clinico, sin estar ayudados por los datos de laboratorio. El diagnóstico definitivo fue, como acontece en todos los casos de embarazos abdominales, motivado por el hallazgo que se hizo durante el acto operatorio. Para hacer más gréfica la descripción, se han tomado algunas lotogratias de la pieza operatoria. 
La historia clínica es la siguiente:

Nombre: T. C. de M. Edad 46 años. Fstado civil: Casada, desde hace 18 cños y de vida genital activa.

Procedencia: Villavicencio. Prolesión: Oticios domésiicos.

Entrada al Hospital: Agosto 29 do 1048 .

Antecedentes familiares: Sin impor:ancia.

Antecedentes patológicos: Enfermeciades de la infancia. Paludismo, Iisentería amibiana. Bronquitis crónica. Antecedentes ginecológicos: Menarc uia a los 15 años, con caracteres norales. Después se instaló un ciclo bien é́inido de $30 \times 3$. Nunca ha presendo thujos vaginales que hagan penit en una posible infección de los exos. Las menstruaciones han sido mpre normales.

ce la paciente que su ciclo sólo se visio interrumpido una vez, y de tio hace ya quince años; en esa épo. tuvo una amenorrea de tres meses, cabo de los cuales sobrevino una trorragia con coágulos abundan'es - duró tres dias y que se vió acorn. lada de sensación de fiebre y de igero dolor en la fosa illaca dereDe entonces para acá su cicio sirual continuó absolutamerte nor-

datos suministrados por la pote y corroborados por el médico inte se sabe que antes y después accidente de la amenorrea y de la trorragic, había sido sometida a tratemiento para su esterilidad.

tas antes de su ingreso al Hospihizo su última consulta en el deseo ser tratada para su bronquitis $y$ ara una sensación de peso que eximentaba en la fosa iliaca derecha.
Ei exmen geveral dió los siguientes datos: Aparato circulatorio: Corazón normal. Pulso 80 Min. T. A. Mx. I60 Mn. 75.

Aparato respiraiorio: Tos húmeda, crónica. Expectoración serosa. Estertores roncantes en ambas bases pulmorares y algunos subcrepitantes en la Lase del pulmón derecho.

Aparato digestivo: Normal. Sólo se encuentra una ligera hipertrotia esplénica.

Senos: Normales.

Examen ginecológico: Tacto vaginai: Vagina amplia y normal. Cuello de 3 cms. de longitud en situación central, de forma y consistencia normales. Oriticio externo cerrado, de nulípara; fondo de saco anterior libre, fondo de saco de Douglas libre; fondo de saco icteral izquierdo libre también; fondo de saco lateral derecho ocupado y no doloroso.

Combinado el tacto con la palpación abdominal se encuentra una masa, cuyos movimientos se transmiten al cuello uterino y que por lo tanto es el útero, de tamaño normal pero cue sobre el cuemo izquierdo se continúa con una tumelacción dura, ligeramente móv.l, no dolorosa y del tamaño de un huevo de gailina; los movimientos imprimidos a esto masa se transmiten aunque no muy nítidamente al cuello. En el fondo de saco icteral derecho se encuentra otra niasa dura, irregular, alargada, no dolorosa, del tamaño suficiente para "lenarlo por completo, poco o nada nóvil e indeperdiente de la matriz.

Con base en este examen se formu15 el siguiente diagnóstico: Fibromio. ina uterino. Quiste ovárico derecho. Ironquitis crónica. 


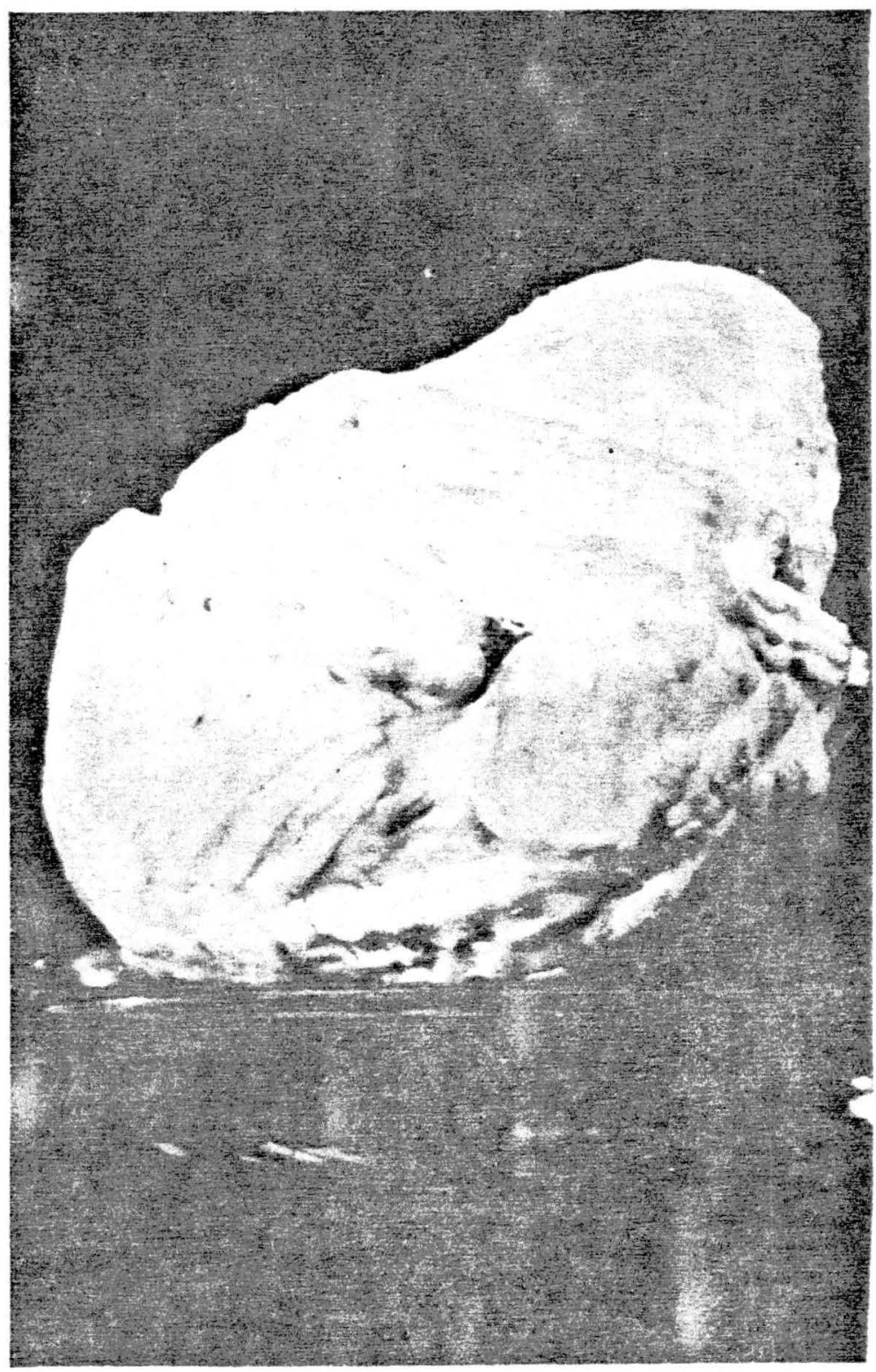

LITOKELIFOSPEDION 
Intervención. Laparotomía mediana infraumbilical de $15 \mathrm{cms}$. más o menos.

Abierta la cavidad abdominal se encontró sobre la fosa ilíaca derecha y desbordándola un poco hacia arriba, el epiplón mayor envolviendo und masa dura que había contraído adherencias con el ciego. Fácilmente tue ciesprendido el epiplón y se vio que lo que se había tomado como un quisite del ovario derecho era un cuerpo de consistencia de piedra, pegado al cual se hallaba el apéndice. Extráído el cuerpo extraño intracibdominal sin diiicultad alguna, se continuó la intervención sobre la matriz. Esta se hallaba casi totalmente alcanzada por un proceso de fibromatosis y del cuerno uterino izquierdo se desprendía un fibroma pediculado. Revisados los anexos de ambos lados se encontraror absolutamente normales.

Ante este cuadro se decidió verificar una histerectomía subtotal, la cual se logró sin inconveniente ninguno para su realización. Por último se procedio a reparar la pared abdominal en la forma acostumbrada y la mujer salió en perfectas condiciones de la sala de cirugía.

Examinada la pieza operatoria se pudo establecer el siguiente diagnóstico quirúrgico: Fibromatosis uterina. Fibromioma Pediculado. Subseroso del Fondo. Litokelifospedión correspondiente, $\alpha$ un embarazo abdominal primitivo de 3 a 4 meses.

Esta enferma parece haber portado un embarazo abdominal primitivo, puesto que su historia no tiene dato de un ictus abdómino-hemorragico indice del fin de una evolución tubaria de su preñez. En efecto, careció ella del dolor ogudo y cunque tuvo algu- na hernorragia externa, ésta fue determinada por la expulsión de la caduca a los tres meses más o menos de amenorrea.

Estos datos clínicos desempeñan un papel primordial en el diagnóstico, kues se podría pensar que el haber encontrado intactos los anexos no excluiría por completo una posible cicatuización de la trompa que despues de 15 años no fuera perceptible. Vale la pena anotar que a la integridad anatomica de las trompas y de los ovarios se unía la falta de síntomas y signos de la ruptura. Podría ser también demostrativo la falta de adherencias del tumor o de su envoltura epiploicr, con los órganos anexiales después de haber tenido el embarazo un crecimiento intratubario seguido de ruptura, las adherencias a dichos órganos habrían sido grandes y persistentes.

El ctro hecho importante es la trans. tomación sufrida por el feto muerto y retenido. Por una parte hay la rare$z a$ de la calcificación de los anexos, pues lo mór frecuente as que ella ce realice solcmente en el feto. For otra parte se confirma que la dicha transformación es la más tavorable para la muier, puesto que sin mayares complicaciones la lleva dentro de su abciomen por espacio de muchos años.

\section{BIBLIOGRAFIA}

\section{Autores nacionales:}

Archila A. Enrique. El embarazo extrauterino. 1946.

Ramirez M. Rafael F. Embarazo Ectópico casi a término Revista Medicina y Cirugía. 1946. 
Ramírez M. Rafael F. Un caso más de embarazo ovárico llegado a término. Revista Médica, órgano de la Academia Nacional de Medicina.

Serpa Roberto. Embarazo extrauterino. Revista de la Facultad Nacional de Medicina. 1940.

Autores extranjeros:

Calatroni Carlos J. Terapéutica Ginecológica $4^{a}$ edición, 1946 .

Calderón Horacio y Molina V. Embarazo abdominal primitivo. Día Médico. 1946.

Charpentier A. Traité de laccouchement.

Conill Víctor. Embarazo ectópico. 2" edición, 1945.

rossen and Crossen. Diseases of women. ga edición.

urtis Arthur Hale. Ginecología. 1941
Davis Carl Henry. Ginecology and C. steirics. 1946.

Fabre. Manual de Obstetricia. 1941.

Coldberger Morris A. Ginecology surgery. 1942.

Hardy John A. Sinopsis of the diagnosis of the surgical diseases of the abdomen. $2^{2}$ edic., 1945.

Maygriery Schwabb. Manual de Obstetricia. 1943.

Novak. Ginecología. 1945.

Faradelo Carlos A. La histerosalpingografía en el diagnóstico del embarapo ectópico abdominal.

Pérez Manuel Luis. Tratado de obstetricia. 1943.

Recassens Girol. Tratado de Obstetricia.

Studdiford William E. Primary Peritoneal Pregnancy. An J. Obst. and Gin. Vol. 44,1942

\title{
Consideraciones sobre rupturas uterinas durante el año de 1949
}

\author{
Por EUDORO CASTIILO VEGA \\ Jefe de Clínica de Matemidad.
}

Ina de las complicaciones mús scrias que se pueden presentar en el e ercicio de la tocología es la ruptura i. erina.

Este accidente puede acontecer en el curso del embarazo o en el momento del trabajo; puede ser accidental $y$ raras veces espontáneo, pero casi simpre obedece a causa traumática. In la primera mitad del embarazo puede ser ocasionado por una gestación en la porción intersticial de la trompa, en un útero bicomio, en un útero in- fantil y en otras veces por excesiva invasión de la pared uterina por los elementos embrionarios, Al final de la gestación, el accidente por io general, está relacionado en los casos espontáneos, por la presencia de tejidos cicatrizales en la pared uterina, odelgazados progresivamente por la distensión creciente del órgano. Poroschin las ha atribuído a la ausencia relativa del tejido elástico; Jellinghaus y otros autores creen en la debilidad intrínseca de la pared uterina, por la 